\title{
Automated measurement of unsaturated iron binding capacity is an effective screening strategy for C282Y homozygous haemochromatosis
}

P E Hickman, L F Hourigan, L W Powell, F Cordingley, G Dimeski, B Ormiston, J Shaw, W Ferguson, M Johnson, J Ascough, K McDonell, A Pink, D H G Crawford

\begin{abstract}
Background-C282Y hereditary haemochromatosis is an appropriate condition for population screening. Transferrin saturation, the best screening test to date, is relatively expensive, labour intensive, and cannot be automated. Unsaturated iron binding capacity is a surrogate marker of transferrin saturation and its measurement can be automated.

Aims-To evaluate a screening strategy for C282Y hereditary haemochromatosis in a tertiary hospital environment based on unsaturated iron binding capacity as the initial screening test.

Methods-Measurement of unsaturated iron binding capacity was adapted to the main laboratory analyser. An unsaturated iron binding capacity of less than $30 \mu \mathrm{mol} / 1$ was identified as an appropriate decision point and 5182 consecutive subjects were screened over 28 consecutive days.

Results-Of those screened, 697 had an unsaturated iron binding capacity less than $30 \mu \mathrm{mol} / 1$. Of these, transferrin saturation was greater than $40 \%$ in 294 . A total of 227 were able to be genotyped for the C282Y mutation. Nine subjects homozygous for C282Y were identified. Based on full cost recovery, affected persons were identified at a cost of Aus $\$ 2268.77$ per case (approximately US\$1496).

Conclusion-Automated measurement of unsaturated iron binding capacity enables a cost effective, large scale population screening programme for $\mathrm{C} 282 \mathrm{Y}$ hereditary haemochromatosis to be developed. (Gut 2000;46:405-409)
\end{abstract}

Keywords: unsaturated iron binding capacity; haemochromatosis; screening

Hereditary haemochromatosis (HHC) is one of the most common autosomal recessive diseases in whites. The reported prevalence of the condition in this population is approximately $0.3 \%,{ }^{1}$ and may be as high as $1.0 \%$ in populations of Celtic origin. ${ }^{2}$ It has been suggested that this high prevalence and the widespread availability of an acceptable and effective treatment make haemochromatosis an ideal condition for widespread population screening programmes. Indeed, population screening for $\mathrm{HHC}$ is an area of intense interest and a subject of some controversy as haemochromatosis fulfils almost all the criteria for screening programmes as defined by the World Health Organisation. ${ }^{3}$ However, the natural history of the disease is incompletely understood, and there is vigorous debate regarding optimal screening strategies for this condition. The best phenotypic marker for the condition seems to be transferrin saturation. ${ }^{4}$ However, the measurement of transferrin saturation is not automated, is labour intensive, and relatively expensive; these factors preclude long term screening programmes based on this test. Unsaturated iron binding capacity (UIBC) is a surrogate marker of transferrin saturation and has been reported to identify $100 \%$ of iron overloaded patients and $95 \%$ of patients with normal iron stores. ${ }^{5}$ It has been proposed as a suitable screening method for HHC. ${ }^{6-8}$

In 1996, Feder et al described a novel gene (now known as HFE) located on chromosome 6. Eighty two per cent of their American patients with hepatic iron overload had a substitution of cysteine to tyrosine at position 282 of the gene product. ${ }^{9}$ Since this publication it has become apparent that in populations of a northern European extraction, most primary iron overload is associated with the C282Y mutation..$^{10-12}$ An absolute requirement for establishing the diagnosis of expressing HHC is iron overload, which is identified universally by the presence of an increased transferrin saturation. However, not all C282Y homozygotes have biochemical markers of iron overload. ${ }^{13}$ Thus it seems logical to consider patients with C282Y homozygosity and increased transferrin saturation as expressing the disease and the population that should be targeted for detection in a screening programme.

In this study, we employed a strategy in a hospital based screening programme for expressed C282Y homozygous haemochromatosis whereby UIBC was used as the primary screening tool followed by C282Y genotyping in patients with a confirmed elevation of transferrin saturation. We report the clinical and economic efficiency of this strategy in a sample of 5182 consecutive patients at a major Australian tertiary hospital.

Materials and methods

SUBJECTS AND STUDY DESIGN

The study was performed over a four week period in November and December 1997, during which time any blood sample accompanied

Abbreviations used in this paper: HHC, hereditary haemochromatosis; UIBC, unsaturated iron binding capacity. 


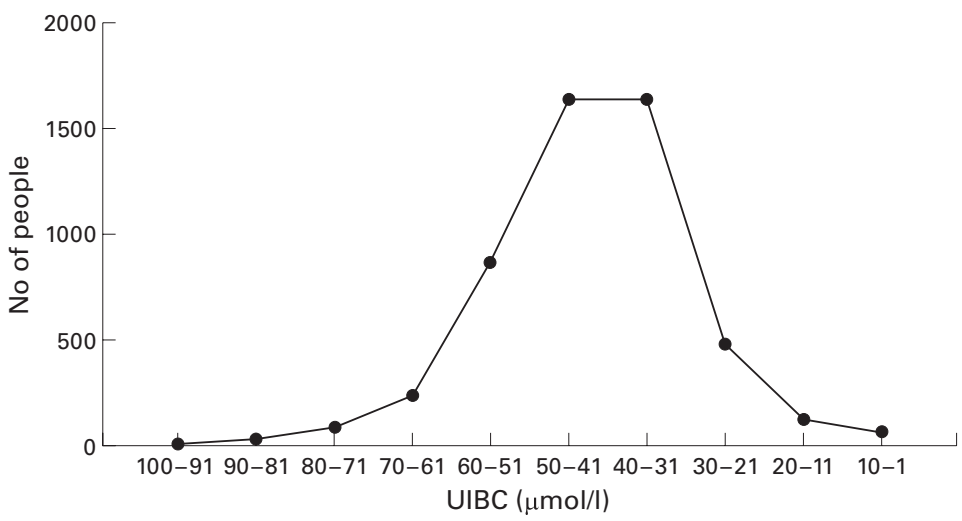

Figure 1 Distribution of unsaturated iron binding capacity (UIBC) in 5182 subjects in a major tertiary hospital.

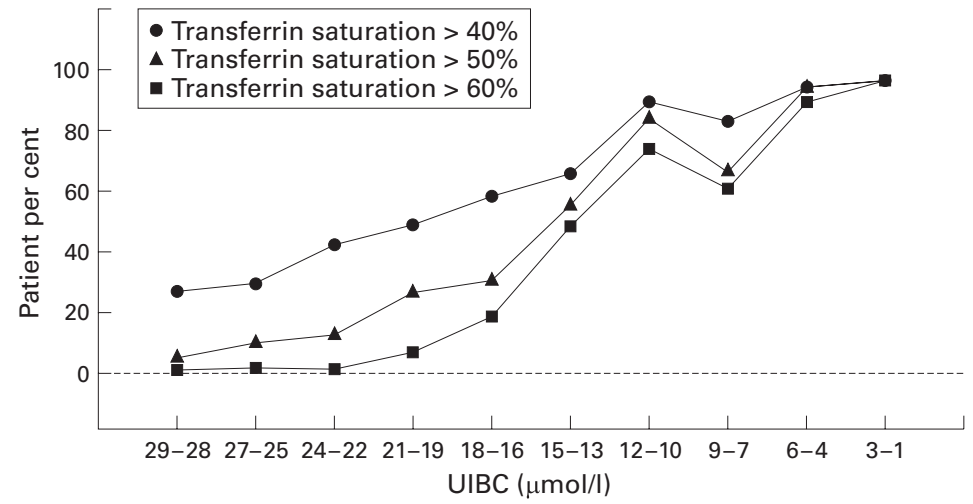

Figure 2 Relation of unsaturated iron binding capacity (UIBC) to transferrin saturation. At each concentration of UIBC less than 30 umol/l, the proportion of patient results with transferrin saturation greater than $40 \%, 50 \%$, and $60 \%$ was calculated.

by a request for analysis on the main laboratory analyser was included in the study. Over the four weeks of the study, 5182 separate persons had plasma samples analysed for UIBC measurement. A preliminary study to determine an appropriate cut off value for UIBC was performed with 73 samples that had a transferrin saturation of $40 \%$ or greater. Regression analysis indicated that a transferrin saturation of $40 \%$ corresponded to a UIBC of $25 \mu \mathrm{mol} / 1$ $(r=-0.87)$. A UIBC cut off value of $30 \mu \mathrm{mol} / 1$ was selected to ensure maximum detection of affected subjects.

If UIBC was less than $30 \mu \mathrm{mol} / \mathrm{l}$, the transferrin saturation was measured on the serum sample; if transferrin saturation was greater than $40 \%$, serum ferritin concentration was measured and genotyping for the $\mathrm{C} 282 \mathrm{Y}$ mutation was performed.

\section{LABORATORY PROCEDURES}

Unsaturated iron binding capacity

The following method was adapted to the Hitachi 747 analyser (Hitachi, Japan). A fixed amount of exogenous iron was added to a sample of plasma to fill all available iron binding sites on transferrin, with a small excess remaining free in plasma. After equilibration, the iron specific dye ferrozine was added, to react with the free iron. The colour that developed was proportional to the initial iron saturation of the transferrin molecule. At a UIBC of $17 \mu \mathrm{mol} / 1$, the interassay coefficient of variation was $3.8 \%$.
Transferrin saturation and ferritin analysis

Transferrin saturation and serum ferritin were measured by standard analytical procedures on a Hitachi 912 analyser (Hitachi, Japan).

\section{Computing}

To prevent repeated assay of the same person during the course of this study, the computer program controlling the laboratory information system was adjusted so that whenever a new sample was presented to the analyser for analysis, a check was performed to see whether previous measurement of UIBC had been made for that patient. If measurement had not been performed, then UIBC was added to the request. This result was not reported but was placed into a separate file which could be accessed at will.

\section{Genotyping}

DNA was extracted from peripheral blood lymphocytes, and the $\mathrm{C} 282 \mathrm{Y}$ mutation was determined by SnaB1 restriction enzyme digestion, followed by polymerase chain reaction (PCR) and agarose gel electrophoresis.

\section{Calculation of costs}

All procedures within our laboratory have been costed as part of an Australia wide benchmarking process, and these costs were used to determine the real costs of all procedures used.

ETHICAL APPROVAL

The study was conducted with the approval of the Ethics Committee of the Princess Alexandra Hospital, Brisbane.

\section{Results}

Over the course of the study, UIBC was measured in 5182 patients. Figure 1 shows the distribution of UIBC in this hospital population. Figure 2 shows the relation between UIBC and the transferrin saturation. Some patients with a transferrin saturation greater than $40 \%$ had a UIBC greater than $30 \mu \mathrm{mol} / 1$; however, essentially all patients with transferrin saturation greater than $50 \%$ were detected with this UIBC value, and all with a transferrin saturation greater than $60 \%$.

A total of 697 of the 5182 subjects $(13.5 \%)$ had a UIBC less than $30 \mu \mathrm{mol} / 1$. The transferrin saturation was greater than $40 \%$ in 294 of these 697 subjects $(42.2 \%)$; of these 294, genotyping for HFE mutations was able to be conducted in 227 (67 had either died or were lost to follow up). Initially we genotyped only those persons with no apparent cause for an increased transferrin saturation. Of 46 persons in this category we found two $(4.34 \%)$ to be homozygous for the C282Y mutation. We decided to extend the genotyping to all persons with increased transferrin saturation regardless of cause. Of the 227 patients finally genotyped, nine $(3.96 \%)$ were homozygous for the C282Y mutation, 44 were heterozygous, and 174 were homozygous normal.

Table 1 shows the iron related studies of the nine subjects with C282Y HHC. Two of these subjects had a previous diagnosis of expressed HHC as judged by histological and quantitative 
Table 1 Iron studies in homozygotes for the C282Y mutation

\begin{tabular}{lllllc}
\hline Case no & Sex & Age $(y)$ & UIBC $(\mu \mathrm{mol} / \mathrm{l})$ & $\begin{array}{l}\text { Transferrin } \\
\text { saturation }(\%)\end{array}$ & Ferritin $(\mu \mathrm{g} / \mathrm{l})$ \\
\hline 1 & $\mathrm{M}$ & 22 & 2 & 0.97 & 836 \\
2 & $\mathrm{M}$ & 43 & 3 & 0.90 & 804 \\
3 & $\mathrm{M}$ & 53 & 9 & 0.77 & 1088 \\
4 & $\mathrm{M}^{\star}$ & 67 & 10 & 0.76 & 416 \\
5 & $\mathrm{~F}$ & 36 & 16 & 0.72 & 156 \\
6 & $\mathrm{~F}$ & 29 & 18 & 0.58 & 145 \\
7 & $\mathrm{M}^{\star}$ & 81 & 24 & 0.47 & 328 \\
8 & $\mathrm{~F}$ & 56 & 25 & 0.51 & 96 \\
9 & $\mathrm{~F}$ & 79 & 26 & 0.42 & \\
\hline
\end{tabular}

^Haemochromatosis known. UIBC, unsaturated iron binding capacity.

assessment of body iron stores, and quantitative phlebotomy. The diagnosis was a new finding in the remaining seven patients. None of the nine patients came from gastroenterology wards or clinics. Based on full cost recovery, the total cost of this study was Aus $\$ 20418.90$ (US\$1347, using Aus $\$ 1.00=$ US\$0.66). Therefore, the average cost per case detected was Aus $\$ 2268.77$, which is approximately US\$1496.

\section{Discussion}

The natural history of HHC is incompletely understood but the condition is characterised by a long latent period between onset of disease and the development of hepatic cirrhosis, during which time affected subjects are often asymptomatic. Venesection therapy is an acceptable treatment which prolongs life if instituted before the development of cirrhosis. ${ }^{14}$ These are important criteria endorsing the desirability of developing a widespread population screening programme for HHC in populations at risk. In this study, we showed the clinical and economic utility of unsaturated iron binding capacity as a screening tool for C282Y HHC in a hospital population. The results indicate that the actual cost of detecting each case was Aus $\$ 2268.77$ (US $\$ 1496$ ). This compares very favourably with other screening programmes which are currently in use or being advocated. For example, to save one year of life by mammographic screening for breast cancer in women over 50 years of age costs $\$ 21400$. $^{15}$ Similarly, to save one year of life by screening for colorectal cancer using haemoccult testing of faeces costs $\$ 20000-30000 .{ }^{16}$ The family members of the probands identified in this study have not, as yet, been screened for iron overload. The identification of affected family members will reduce the overall cost per case detected even further because the major cost in a screening programme for HHC is the expense associated with the initial screening step. ${ }^{17}$ This study complements the recent findings of Adams et al who recently screened 5496 healthy blood donors and showed that UIBC was an excellent screening tool in that population. ${ }^{8}$ The current study extends those observations and shows that screening for C282Y HHC with UIBC can be successfully applied in a major tertiary hospital setting.

In 1996, Feder et al described a novel gene (now known as HFE) located on chromosome 6. Eighty two per cent of their American haemochromatosis patients had a substitution of cysteine to tyrosine at position 282 of the gene product ${ }^{9}$ and on close clinical evaluation, a significant proportion of subjects with apparent hereditary haemochromatosis had secondary causes of iron overload, ${ }^{18}$ indicating that the association of $\mathrm{C} 282 \mathrm{Y}$ and HHC is probably understated. Subsequently, it was proposed that screening programmes based on C282Y genotype analysis would be appropriate. However, we have recently shown that up to $30 \%$ of women and $10 \%$ of men homozygous for this mutation may not show evidence of phenotypic expression. ${ }^{13}$ Non-expression of an underlying genetic defect has important implications for insurance, vocational, and financial matters, and the demonstrated rate of non-expression clearly limits the use of a widespread screening programme based on genotype alone.

The screening strategy employed in the current study may identify subjects with limited disease expression (raised transferrin saturation but normal serum ferritin concentration and homozygosity for the C282Y mutation; see case 9, table 1). However, our evidence indicates that subjects with these phenotypic characteristics are not common and occur in approximately $11 \%$ of the subjects detected by the screening strategy. Recently, an expert panel reached a consensus view stating that phenotypic measurements, rather than genetic testing, is recommended for population based screening for haemochromatosis. ${ }^{19}$ Most authors agree that transferrin saturation is the best screening test for haemochromatosis. ${ }^{4}$ However, it is difficult to apply a large screening strategy for haemochromatosis based on transferrin saturation because its measurement cannot be readily automated and demand would overwhelm routine pathology laboratories. The data in the present study show that: UIBC is a satisfactory surrogate for transferrin saturation; its measurement can be automated; and application of a screening strategy based on UIBC can be successfully applied to a hospital based population in a cost effective manner.

The reference range for UIBC provided by the manufacturer was greater than $20 \mu \mathrm{mol} / 1$. A preliminary analysis indicated that a UIBC of $20 \mu \mathrm{mol} / 1$ corresponded to a transferrin saturation of approximately $55 \%$. There have been several reports of the most appropriate "cut off" value of transferrin saturation for screening for haemochromatosis. Dadone and colleagues ${ }^{20}$ suggested a value of $62 \%$ whereas Leggett and colleagues ${ }^{1}$ suggested a value of $55 \%$. There is now convincing evidence that some subjects with haemochromatosis have a transferrin saturation lower than these values. Recent mathematical modelling of data from a large Queensland population showed that a transferrin saturation of $45 \%$ would identify $98 \%$ of homozygotes of both sexes. ${ }^{21}$ Thus, to provide a reasonable safety margin and based on conclusions from a preliminary study, the UIBC decision point was extended to 30 $\mu \mathrm{mol} / 1$. Data presented in fig 2 indicate that even with this extended value, some persons with a transferrin saturation of between $40 \%$ and $50 \%$ may not be detected with a UIBC of $30 \mu \mathrm{mol} / 1$. However, as the transferrin satura- 
tion increased to more pathological values (above 50\%), the proportion of subjects with an increased transferrin saturation who were not detected by a UIBC of less than $30 \mu \mathrm{mol} / 1$ fell substantially. Crawford and colleagues ${ }^{13}$ recently studied subjects homozygous for the C282Y mutation in the HFE and their data indicated that a transferrin saturation value of $50 \%$ would identify more than $90 \%$ of affected males and $80 \%$ of affected females. Clearly, screening programmes cannot identify $100 \%$ of all affected subjects as outlay costs to achieve such a result would be prohibitive.

Hereditary haemochromatosis is largely a disease of northern European populations with a reported prevalence of 1:300-1:400. ${ }^{1}$ The prevalence of haemochromatosis in the population studied in this hospital was 1:575. There are a number of reasons why the prevalence in the current study was less than expected. Fifteen per cent of subjects in the study were Asian or other ethnic grouping unassociated with C282Y. ${ }^{22}$ Furthermore, only $77.2 \%$ of the 294 subjects with a UIBC of less than $30 \mu \mathrm{mol} / 1$ were genotyped. This resulted either from patient death or loss to follow up in 67 subjects. The failure to perform genotype analysis in all subjects reflects the difficulties that will be encountered when this screening strategy is employed in a true clinical setting, as some subjects will choose not to pursue complete evaluation and others will not be contactable because of itinerant lifestyles. The rate of detecting $\mathrm{C} 282 \mathrm{Y}$ homozygotes was remarkably constant as subjects were recalled for genotyping. We predicted that if all 294 subjects were genotyped, $11.65 \mathrm{C} 282 \mathrm{Y}$ homozygotes would be identified. If the subjects from ethnic groupings unassociated with C282Y are excluded from the analysis, this would provide a prevalence of approximately 1:380 which is similar to that found by Leggett et al. ${ }^{1}$

In addition to identifying affected haemochromatosis subjects, the implementation of a large scale screening programme using UIBC offers two other advantages. Firstly, a high UIBC occurs in iron deficiency and the widespread use of UIBC may result in earlier identification of subjects with colorectal cancer, peptic ulcer disease, and malabsorption. Secondly, the treatment of affected subjects by venesection and the use of this blood for transfusion purposes would help overcome the shortage of blood supplies which is a frequent occurrence in Australia and other countries. Blood transfusion policies in Australia are changing and blood from haemochromatosis subjects is now considered suitable for transfusion purposes.

Our experience of which persons to genotype is informative. In this large tertiary hospital, many patients have reasons other than HHC for an increased transferrin saturation, for example, transfusion dependency. Interestingly, the rate of detecting homozygotes was similar in those with, and those without, clinical reasons for increased transferrin saturation. This observation suggests that completing the proposed screening strategy is important for subjects irrespective of whether there is an apparent underlying clinical reason for the increased transferrin saturation.

This study was not designed to test the sensitivity or specificity of UIBC. Rather, the study was performed to test the economic efficiency of UIBC as the initial screening tool for C282Y haemochromatosis. However, it should be noted that almost $60 \%$ of those patients with a UIBC of less than $30 \mu \mathrm{mol} / 1$ did not have a transferrin saturation greater than $40 \%$. In the preliminary regression analysis, a UIBC of 25 $\mu \mathrm{mol} / 1$ corresponded to a transferrin saturation of $40 \%$. As previously explained, a UIBC of less than $30 \mu \mathrm{mol} / 1$ was selected as the critical value to ensure maximum detection of affected subjects. A combination of error associated with regression analysis and the fact that $24 \%$ of subjects with a UIBC less than $30 \mu \mathrm{mol} / 1 \mathrm{had}$ a UIBC between 25 and $30 \mu \mathrm{mol} / 1$ explains, in part, the apparent poor relation of the UIBC and transferrin saturation near the cut off value of $30 \mu \mathrm{mol} / \mathrm{l}$. Determining the precise cut off value to maximise efficiency of the UIBC screening test is the subject of the current study.

This study shows that the capacity now exists for large scale population screening for C282Y HHC based on automated measurements of unsaturated iron binding capacity. Screening, even in a major hospital setting is clinically and cost effective. Furthermore, the cost per case detected is much less than other disease processes for which large scale population screening already exists. Our data suggest that automated measurement of UIBC is an appropriate strategy for large scale screening programmes for C282Y HHC in hospital settings.

We would like to acknowledge the assistance of Roche Diagnostics in the conduct of this study.

1 Leggett BA, Halliday JW, Brown NN, et al. Prevalence of haemochromatosis amongst asymptomatic Australians. $\mathrm{Br}$ haemochromatosis amongst

2 Smith BN, Kantrowitz W, Grace ND, et al. Prevalence of hereditary hemochromatosis in a Massachusetts corporation: is Celtic origin a risk factor? Hepatology 1997; 25:1439-46

3 Wilson JMG, Jungner G. Principles and practice of screening for disease. Public Health Papers WHO 1968;34: 26-39.

4 Bassett ML, Halliday JW, Bryant S, et al. Screening for hemochromatosis. Ann NY Acad Sci 1989;526: 274-8.

5 Pintar J, Skikne BS, Cook JD. A screening test for assessing iron status. Blood 1981;59:110-13.

6 Witte DL, Crosby WH, Edwards CQ, et al. Practice guideline development task force of the College of American Pathologists. Hereditary hemochromatosis. Clin Chim Acta 1996;245:139-200.

7 Skikne BS, Cook JD. Screening test for iron overload. Am $\mathcal{F}$ Skikne BS, Cook JD. Scre
Clin Nutr 1987;46:840-3.

8 Adams PC, Keretz AE, Barr R, et al. Population screening for haemochromatosis with the unbound iron capacity [abstract]. Gastroenterology 1998;114:A1199.

9 Feder JN, Gnirke A, Thomas W, et al. A novel class 1-like gene is mutated in patients with hereditary haemochromatosis. Nat Genet 1996;13:399-408.

10 Jazwinska EC, Cullen LM, Busfield F, et al. Haemochromatosis and HLA-H. Nat Genet 1996;14:249-51.

11 Jouanolle AM, Gandon G, JezequeL P, et al. Haemochromatosis and HLA-H. Nat Genet 1996;14:251-2.

12 Beutler E, Gelbart T, West C, et al. Mutation analysis in hereditary hemochromatosis. Blood Cell Mol Dis 1996;22: 187-94.

13 Crawford DHG, Jazwinska EC, Cullen LM, et al. Expression of HLA-linked hemochromatosis in subjects homozygous or heterozygous for the C282Y mutation. Gastroenterology 1998;114:1003-8. 
14 Niederau C, Fischer R, Purshel A, et al. Long term survival in patients with hereditary hemochromatosis. Gastroenterolin patients with hereditary
ogy $1996 ; 110 ; 1107-9$

15 Salzmann P, Kerlikowske K, Phillils K. Cost-effectiveness of extending screening mammography guidelines to include women 40 to 49 years of age. Ann Intern Med 1997;27:95565.

16 Simon B, Fletcher RH. Should all people over the age of 50 have regular fecal occult-blood tests? $N$ Engl f $\mathrm{Med}$ 1998;338:1151-5.

17 Bassett ML, Leggett BA, Halliday JW, et al. Analysis of the cost of population screening for haemochromatosis usin biochemical and genetic markers. F Hepatol 1997;27:517-24

18 Shaheen NJ, Bacon BR, Grimm IS. Clinical characteristics of hereditary HC patients who lack the C282Y mutation. Hepatology 1998;28:526-9.
19 Burke W, Thomson E, Khoury MJ, et al. Hereditary hemochromatosis: gene discovery and its implicationsfor population-based screening. $\quad \exists A M A \quad$ 1998;280: $172-8$.

20 Dadone MM, Kushner JP, Edwards CQ, et al. Hereditary hemochromatosis. Analysis of laboratory expression of the diseases by genotype in 18 pedigrees. Am 7 Clin Pathol 1982;78:196-207.

21 McLaren CE, McLachlan GJ, Halliday JW, et al. Distribution of transferrin saturation in an Australian population: relevance to the early diagnosis of hemochromatosis. Gastroenterology 1998;114:543-9.

22 Merryweather-Clarke AT, Pointon JJ, Shearman JD, et al. Global prevalence of putative haemochromatosis mutations. F Med Genet 1997;34:275-8. 